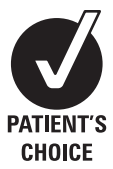

See Editorial commentary, p 1065

${ }^{1}$ Cambridge Centre for Brain Repair, University of Cambridge Cambridge, UK

Institute of Neurology, University College London, London, UK

${ }^{3}$ Institute of Public Health University of Cambridge,

Cambridge, UK

${ }^{4}$ Department of Experimental

Psychology, University of Cambridge, Cambridge, UK

Correspondence to

Dr J R Evans, Cambridge Centre for Brain Repair, University of Cambridge, Forvie Site,

Robinson Way, Cambridge CB2

OSD, UK;

jonathanevans@doctors.org.uk

Received 4 January 2011

Revised 9 March 2011

Accepted 4 April 2011

Published Online First

18 May 2011

\title{
The natural history of treated Parkinson's disease in an incident, community based cohort
}

\author{
Jonathan R Evans, ${ }^{1}$ Sarah L Mason, ${ }^{1}$ Caroline H Williams-Gray, ${ }^{1}$ Thomas Foltynie, ${ }^{2}$ \\ Carol Brayne, ${ }^{3}$ Trevor W Robbins, ${ }^{4}$ Roger A Barker ${ }^{1}$
}

\section{ABSTRACT}

Background Our understanding of the natural history of idiopathic Parkinson's disease (PD) remains limited. In the era of potential disease modifying therapies, there is an urgent need for studies assessing the natural evolution of treated PD from onset so that relevant outcome measures can be identified for clinical trials. No previous studies have charted progression in unselected patients followed from the point of diagnosis. Methods A representative cohort of 132 PD patients was followed from diagnosis for up to 7.9 years (mean 5.2 years). Comprehensive clinical and neuropsychological evaluations were performed every 18 months. Disease progression was evaluated using well validated clinical measures (motor progression and development of dyskinesia on the Unified PD Rating Scale and Hoehn-Yahr scale, dementia onset according to DSM-IV criteria). Multi-level linear modelling was used to chart the nature and rate of progression in parkinsonian symptoms and signs over time. The prognostic importance of baseline demographic, clinical and genetic variables was evaluated using survival analysis.

Results Axial (gait and postural) symptoms evolve more rapidly than other motor features of PD and appear to be the best index of disease progression. Conversely, conventional outcome measures are relatively insensitive to change over time. Earlier onset of postural instability (Hoehn-Yahr stage 3) is strongly associated with increased age at disease onset and has a significant impact on quality of life.

Conclusions Dementia risk is associated with increased age, impaired baseline semantic fluency and the MAPT $\mathrm{H} 1 / \mathrm{H} 1$ genotype. The efficacy of disease modifying therapies may be more meaningfully assessed in terms of their effects in delaying the major milestones of PD, such as postural instability and dementia, since it is these that have the greatest impact on patients.

\section{INTRODUCTION}

Parkinson's disease (PD) is a progressive neurodegenerative disorder of unknown aetiology for which there is no known cure. While symptomatic treatments can ameliorate the core, motor symptoms, we are increasingly looking to deliver therapies to modify the underlying course of the disease. The effectiveness of symptomatic treatment makes it difficult to study the relationship between pathological progression, which we know to be inexorable, and the clinical evolution of the disease: the natural history of treated PD remains poorly characterised.

Improving our understanding of the progression of $\mathrm{PD}$ in the era of effective symptomatic treatment is important for a number of reasons. It will aid in prognostication and will also allow the clinical effects of treatment in individual patients to be evaluated against a meaningful baseline. Most topically, understanding progression in $\mathrm{PD}$ enables the identification of relevant outcome measures for clinical trials of putative disease modifying agents.

Surprisingly little has been written about the natural evolution of symptoms and signs in $\mathrm{PD}$. Studies from the pretreatment era were not conducted systematically and have proved largely uninformative, and more recent natural history studies have all tended to have short follow-up periods and involve highly selected patients, such as those in placebo arms of clinical trials, ${ }^{1-3}$ clinic based evaluations in secondary or tertiary care $e^{4-6}$ or, where population based cohorts have been studied, recruited patients of varying disease duration. ${ }^{78}$ There are no previous naturalistic studies in $\mathrm{PD}$ using unselected, representative patients followed from diagnosis, which would most accurately recapitulate the evolution of disease in true to life populations encountered in clinical practice.

There is considerable heterogeneity both in the rate of clinical progression and the risk of disease complications in $\mathrm{PD}^{9}$ but the relative contributions of clinical, genetic and indeed iatrogenic factors to this heterogeneity remain incompletely understood. Our aim in this study was to track the evolution of disease from diagnosis in a representative $\mathrm{PD}$ cohort to investigate which clinical measures of motor progression are most sensitive to change with time, and to identify variables which influence prognosis. This supersedes earlier work in which we have reported the association between age, genotype at the microtubule associated protein $\tau$ gene (MAPT) locus (encoding the $\tau$ protein) and baseline cognitive profile in determining the risk of incident dementia, ${ }^{10-12}$ and we present for the first time our data on motor and non-motor outcomes in an unselected PD population over an extended follow-up period.

\section{METHODS}

\section{Patients}

Subjects were participants in an epidemiological study of incident parkinsonism conducted over a 2 year period between December 2000 and December 2002, full details of which have been published previously. ${ }^{13}$ Briefly, subjects with new onset parkinsonism were identified in the community in the county of Cambridgeshire using multiple methods (referrals from primary and secondary care physicians, healthcare records screening) to ensure maximal case ascertainment. The cohort of cases 
assessed was unselected and therefore population representative. Suitable patients were recruited to longitudinal follow-up, undergoing repeat assessments at 18-24 month intervals for up to 7.9 years.

The study was approved by the local ethics committee, and written informed consent was obtained from all patients according to the Declaration of Helsinki.

\section{Assessments and outcome measures}

The diagnosis of PD was made according to UK Parkinson's Disease Society (UKPDS) Brain Bank criteria. ${ }^{14}$ These criteria were reapplied at the 3.5 year follow-up to minimise clinical misdiagnosis.

Patients were assessed in the 'on' state and their medication dose recorded as the levodopa equivalent dose (LED) using an algorithm adapted from Brodsky and colleagues, ${ }^{15}$ as we have used previously. ${ }^{11}$ Average daily LED for each respondent was estimated by calculating the area under the curve of a graph of LED against time (with any dose changes assumed to take place at the midpoint between assessments) and dividing this by their total follow-up period.

PD motor state was evaluated using two widely used rating scales, the Unified Parkinson's Disease Rating Scale (UPDRS) and the Hoehn-Yahr Scale (HYS). The UPDRS is a comprehensive 44 item scale on which PD symptoms and signs are rated on a ranked, ordinal scale from 0 (absent) to 4 (severe). ${ }^{16}$ The UPDRS has subdivisions, with section 2 (impact of symptoms on activities of daily living) and section 3 (objective assessment of motor signs) often reported separately. Section 3 (hereafter UPDRS-3) has been used frequently as an outcome measure in both clinical trials and naturalistic studies. Section 4 records motor complications, including treatment induced dyskinesias. The UPDRS has undergone extensive clinimetric evaluation and is both consistent and reliable. ${ }^{17}$

Using scores from the UPDRS, patients were classified as tremor dominant, postural instability/gait disorder or intermediate at their initial assessment according to the criteria of Zetusky and colleagues. ${ }^{18}$ The algorithm described by Levy et al was used to derive a subscore for symptoms and signs relatively refractory to medication ('dopa-resistant' symptoms: encompassing scores for speech, posture, gait, postural stability and rising from sitting). ${ }^{19}$ Thus three UPDRS subscores, UPDRS-2 (activities of daily living), UPDRS-3 (motor) and UPDRS 'doparesistant', were defined for each subject at each assessment. In addition, the UPDRS section 4 was used to document the onset of dyskinesias in each subject.

The HYS is a 5 item scale developed in the pre-levodopa era to capture the key elements of progression in PD. ${ }^{20}$ While insensitive, one strength of the HYS is that step increases in stage are generally irrevocable. ${ }^{21}$ In particular, the onset of postural instability that characterises HYS stage 3 (HYS-3) has been reported to herald a more rapid stage of motor progression, and is associated with increased mortality. ${ }^{622}$ We used the modified version, which defines half point ratings. ${ }^{23}$

Dementia was diagnosed according to DSM-IV criteria in subjects with Mini-Mental State Examination (MMSE) score of $\leq 24$, as has been described previously. ${ }^{11}$ Self-reported quality of life (QOL) was assessed with the PDQ-39 questionnaire. ${ }^{24}$

\section{Genotyping}

DNA was extracted from peripheral blood samples using a standard phenol/chloroform method. Genotyping for rs 9468 (MAPT H1/H2 haplotype) was performed using an allelic discrimination assay run on a $7900 \mathrm{HT}$ detection system (Applied Biosystems, Foster City, California, USA).
Statistical analysis

Multi-level linear modelling

Progression in UPDRS subscores was analysed using a mixed effects linear model (MLM). MLM is well suited to the analysis of longitudinal clinical data as it allows the modelling of a situation where repeated measures in the same subject are not independent but correlated. ${ }^{25}$ To minimise bias, only subjects assessed on three or more occasions were included in the MLM analysis.

Model parameters were specified to allow random slopes and intercepts. An autoregressive (heterogeneous) covariance structure was employed; this assumes measurements made at adjacent time points correlate most strongly and is standard for a repeated measures design. ${ }^{26}$ Estimates of the progression on each UPDRS subscore (dependent variable) were derived as the fixed effect regression coefficient against time (independent variable) using a restricted maximum likelihood method. Subscore gradients were compared using the F test, with Bonferroni adjustment for multiple comparisons; higher gradients were presumed to indicate a superior index of progression. The distributions of slope coefficients and residual error terms were analysed to ensure they conformed to a normal distribution.

As individual UPDRS items can only be scored from 0 to 4 , theoretically the scale is insensitive to change beyond this. The proportion of UPDRS items rated ' 4 ' was calculated across all assessments to assess for such ceiling effects.

\section{Survival analysis}

Time of PD diagnosis was defined as $t=0$. Survival to the onset of HYS-3, diagnosis of dementia and onset of dyskinesia were modelled using Kaplan-Meier analysis. If a landmark was reached, time of onset was taken to be at the midpoint of the preceding inter-assessment interval.

Cox regression was used to investigate covariates which might influence the disease course in PD. These included age at diagnosis, sex, average medication dose, presenting motor phenotype and MAPT genotype. For the dementia analysis, baseline measures of cognitive function were also included: IO estimated by the National Adult Reading Test, production of $\mathrm{F}$, A, $\mathrm{S}$ words in $1 \mathrm{~min}$ each (phonemic fluency), animals named in $90 \mathrm{~s}$ (semantic fluency) and constructional ability using the intersecting pentagons from the MMSE, scored 0-2 according to the algorithm of Ala and colleagues. ${ }^{27}$ Variables were initially evaluated in a univariate analysis. Multivariate analysis was then performed using covariates with an unadjusted $p$ value $\leq 0.05$. The proportional hazards assumption was tested by graphical inspection of log minus log plots.

The relationship between HYS and QOL (PDQ-39) was assessed using analysis of covariance to adjust for the effects of age and disease duration. Post hoc comparisons were performed using Tukey tests with Bonferroni correction.

Statistical analysis was performed in SPSS V.16.0.

\section{RESULTS}

\section{Patients}

The flowchart in figure 1 illustrates the case identification process. A total of 132 patients (75 men) consistently met UKPDS Brain Bank criteria for PD and were included in the survival analysis. Mean age at diagnosis was 70.1 (SD 9.8), mean follow-up was 5.2 years (SD 1.6) and average LED was $479 \mathrm{mg} /$ day (SD 292). Mean performance on the MMSE at baseline was 28.9 (0.6). Of these, data from $\geq 3$ assessments was available for 122 patients (70 men) and these cases were also included in the 
Figure 1 Flowchart illustrating the timeline for follow-up of the incident Parkinson's disease (PD) cohort and the process of case identification for the present analysis. +10 patients with $<3$ assessments were included only in the survival analysis. PDBB, PD according to UK Parkinson's Disease Society Brain Bank criteria.

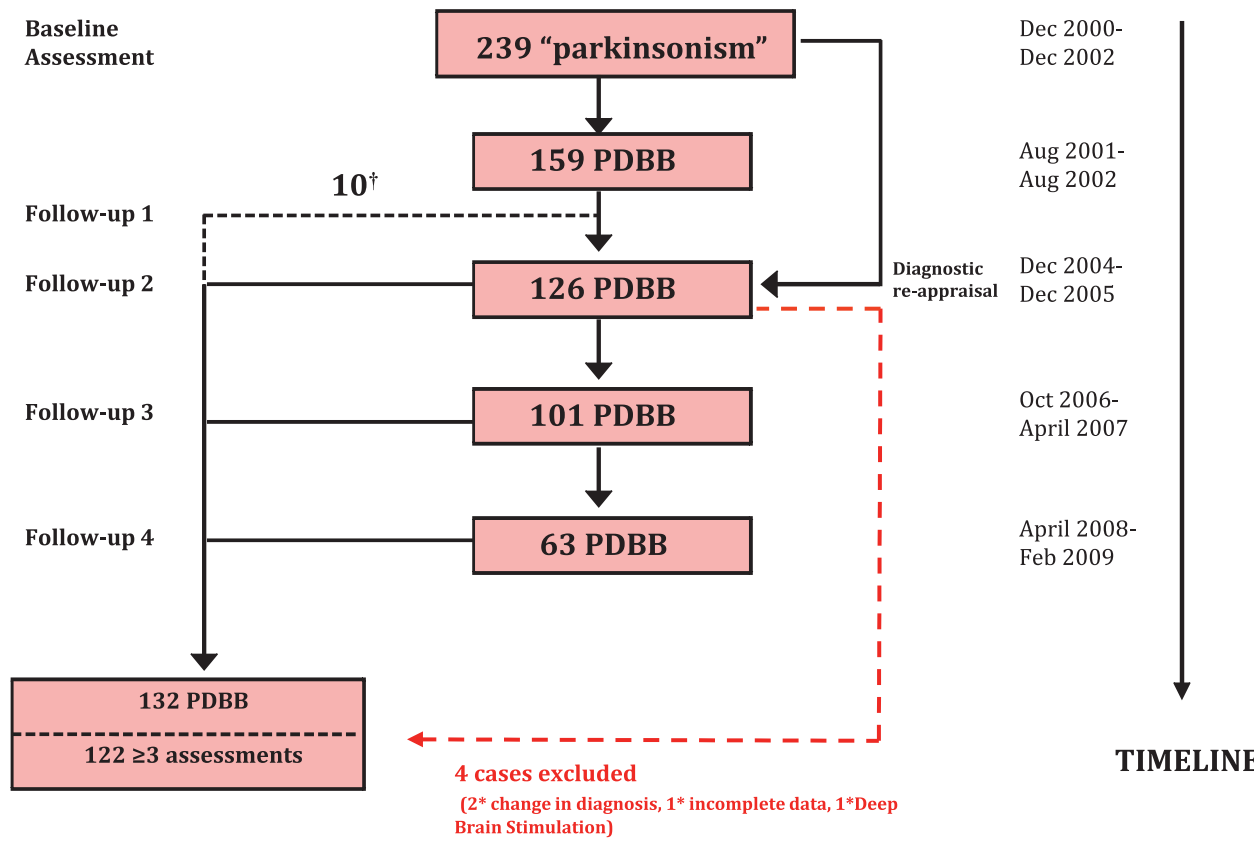

MLM analysis. The equivalent characteristics for this cohort were: mean age 69.5 (9.8), follow-up 5.5 years (1.4), LED $504.9 \mathrm{mg} /$ day (284.2) and baseline MMSE 29.1 (0.7).

\section{Loss to follow-up}

By the fourth round of follow-up, conducted at a mean of 6.6 years from entry, 63/122 (51.6\%) eligible patients remained in the study. Of the remainder, 38 had died, 16 had withdrawn consent (usually due to dementia) and five could not be contacted and were not known to have died. The Standardised Mortality Ratio calculated using the most up to date available lifetable estimates for the UK population was 1.72 (1.16-2.46).

\section{Mixed effects linear model}

Data from 523 assessments in 122 patients were analysed. Only $1.5 \%$ of UPDRS-2 items and $1.2 \%$ of UPDRS items were rated at ceiling (4/4). As over $80 \%$ of these occurred at the latest assessment, ceiling effects are unlikely to have influenced measurement of gradients of change.

Gradients of change for UPDRS subscores are shown in figure 2. Post hoc analysis demonstrated that the gradient for the 'dopa resistant' subscore was significantly greater than that of both the UPDRS-3 ( $\mathrm{F}=-8.250, \mathrm{p}<0.001)$ and the UPDRS-2 $(\mathrm{F}=-5.955, \mathrm{p}<0.001)$. The difference between UPDRS-2 and UPDRS-3 gradients was also significant $(\mathrm{F}=-5.117, \mathrm{p}<0.001)$. Overall mean progression on the UPDRS-3 was 2.24 points/year; on the UPDRS-2, 1.72 points/year. Residual terms generated by the model conformed to a normal distribution.

\section{Survival analysis}

Data from 132 subjects, incorporating 541 assessments and 684 person years of follow-up, was evaluated. Median time to HYS-3 was 3.8 years (range 2.8-4.9) (figure 3A). Less than half of the cohort had developed dementia or dyskinesias by the end of the fifth round of follow-up. The estimated mean time to dementia was 6.2 years (range 5.8-6.3) (figure 3B), and mean time to dyskinesia onset was 6.6 years (range 6.3-7.0) (figure 3C).

The results of Cox regression analysis are shown in table 1 . In the multivariate model, age and a postural instability/gait disorder phenotype at presentation were predictors of more rapid progression to HYS-3, and age, MAPT H1/H1 genotype and impaired semantic fluency at baseline were associated with an increased risk of dementia. The level of dopaminergic therapy was the only covariate associated with increased risk of dyskinesia. A supplementary Kaplan-Meier analysis of the relationship between duration of levodopa therapy and time to dyskinesia onset produced a shorter estimate of 4.8 years (4.4-5.3) although it should be noted that the majority of patients receiving levodopa were co-medicated with a dopamine agonist.

A PDQ-39 score was available for 511/541 assessments (94.5\%). There was a significant positive association between increasing HYS and higher PDQ-39 (worse QOL; F $(7,490)=33.5$, $\mathrm{p}<0.001$, figure 4). Post hoc comparisons revealed that PDQ-39 was significantly increased at HYS-3 relative both to HYS-1

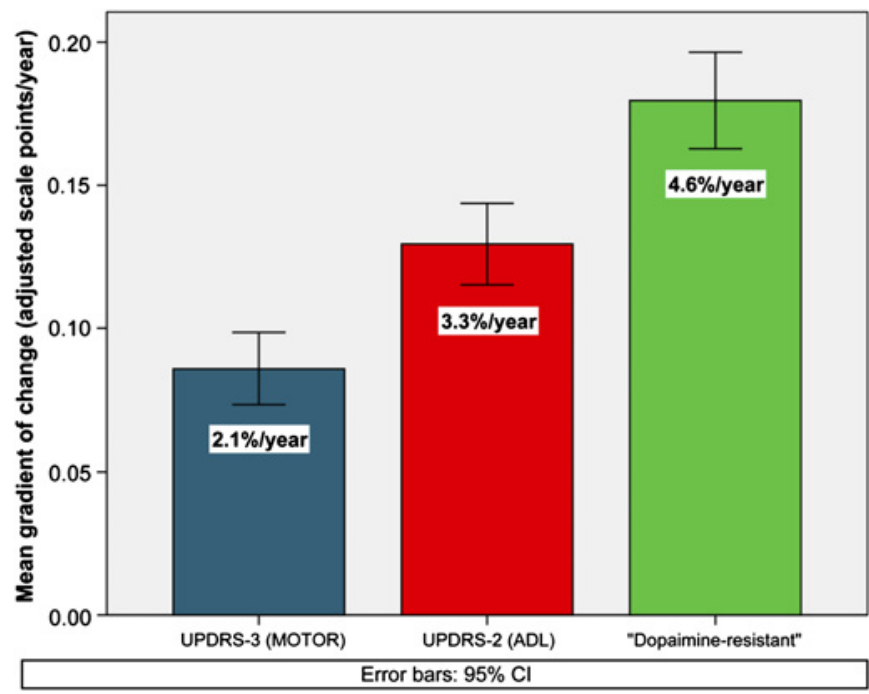

Figure 2 Gradients of change of Unified Parkinson's Disease Rating Scale (UPDRS) subscores (shown as change per item per year) in incident Parkinson's disease. Annual change as a percentage of maximum possible change on each subscore is shown superimposed. $A D L$, activities of daily living. 

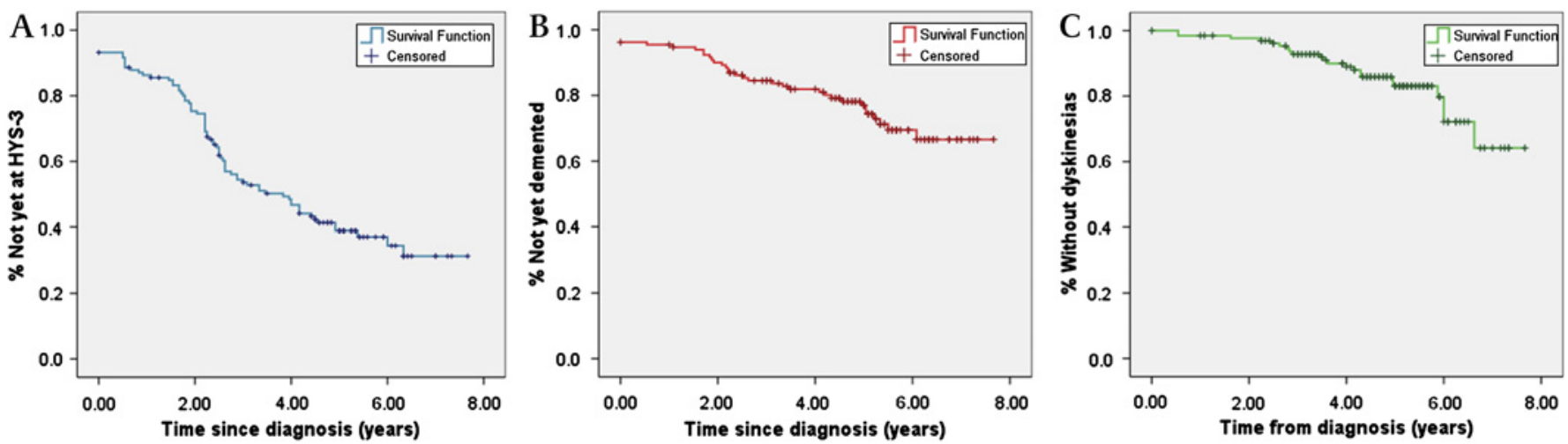

Figure 3 Kaplan-Meier plots of survival to Hoehn-Yahr stage 3 (HYS-3) (A), onset of dementia (B) and onset of treatment induced dyskinesia (C) in the incident cohort.

(unilateral symptoms/signs; $\mathrm{p}<0.001$ ) and HYS-2 (bilateral symptoms/signs without axial involvement; $\mathrm{p}<0.001)$.

\section{DISCUSSION}

This is the first study to report on the rate and pattern of progression of disease in unselected PD patients followed from diagnosis. As our cohort was recruited from the community and followed naturalistically, these results represent the course of PD as it is encountered in true to life clinical settings. The length of follow-up and number of repeated evaluations are also strengths of our study, minimising the measurement error in our estimates of progression and safeguarding against clinical misdiagnosis.

Our results indicate that in treated PD the average annual progression in motor symptoms is modest and equivalent to 2.2 points/year on the UPDRS-3. Estimates of progression rates from previous studies are compared in table 2. Naturalistic

Table 1 Cox regression analysis of predictor variables for progression to Hoehn-Yahr stage 3, dementia and for the development of treatment induced dyskinesias

\begin{tabular}{|c|c|c|c|c|}
\hline Baseline variable & Unadjusted RR (95\% CI) & p Value & Adjusted RR (95\% CI) & p Value \\
\hline \multicolumn{5}{|l|}{ Progression to HYS-3 } \\
\hline Age at diagnosis & $1.069(1.040$ to 1.098$)$ & $<0.001$ & $1.051(1.020$ to 1.084$)$ & 0.001 \\
\hline Sex (M vs F) & $0.903(0.575$ to 1.419$)$ & 0.658 & - & \\
\hline Motor phenotype at presentation $\ddagger$ & & $<0.001$ & & 0.005 \\
\hline TD vs PIGD & $0.379(0.233$ to 0.616$)$ & $<0.001$ & $0.416(0.239$ to 0.724$)$ & 0.002 \\
\hline TD vs INT & $1.101(0.567$ to 2.135$)$ & 0.777 & $0.980(0.457$ to 2.101$)$ & 0.619 \\
\hline Average LED & $0.999(0.979$ to 1.019$)$ & 0.188 & - & - \\
\hline MAPT H1/H1 haplotype & $1.178(0.698$ to 1.990$)$ & 0.539 & - & - \\
\hline \multicolumn{5}{|l|}{ Progression to dementia } \\
\hline Age at diagnosis & $1.120(1.068$ to 1.175$)$ & $<0.001$ & $1.123(1.056$ to 1.194$)$ & $<0.001$ \\
\hline Sex (M vs F) & $0.895(0.452$ to 1.773$)$ & 0.751 & - & \\
\hline Motor phenotype at presentation $\ddagger$ & & 0.035 & & 0.606 \\
\hline TD vs PIGD & $0.412(0.193$ to 0.880$)$ & 0.022 & $0.609(0.229$ to 1.617$)$ & 0.319 \\
\hline TD vs INT & $1.130(0.460$ to 2.773$)$ & 0.790 & $0.805(0.264$ to 2.454$)$ & 0.703 \\
\hline MAPT H1/H1 haplotype & $3.992(1.375$ to 11.590$)$ & 0.011 & $4.585(1.457$ to 14.430$)$ & 0.008 \\
\hline Average LED & $0.997(0.977$ to 1.016$)$ & 0.269 & - & - \\
\hline Verbal $10^{*}$ & 0.977 (0.968 to 0.986$)$ & $<0.001$ & $0.984(0.943$ to 1.025$)$ & 0.435 \\
\hline Phonemic fluency $(\leq 31) \dagger$ & 7.314 (2.802 to 19.094$)$ & $<0.001$ & $2.296(0.741$ to 7.118$)$ & 0.150 \\
\hline Semantic fluency $(\leq 19) \dagger$ & 7.886 (2.990 to 20.799$)$ & $<0.001$ & $3.686(1.071$ to 12.690$)$ & 0.039 \\
\hline Pentagon copying & & $<0.001$ & & 0.537 \\
\hline 0 vs 1 & 3.279 (1.413 to 7.609$)$ & 0.006 & $1.051(0.313$ to 3.532$)$ & 0.936 \\
\hline 0 vs 2 & $13.472(5.67$ to 32.00$)$ & $<0.001$ & $2.001(0.553$ to 7.240$)$ & 0.291 \\
\hline \multicolumn{5}{|l|}{ Progression to dyskinesia } \\
\hline Age at diagnosis & $0.981(0.938$ to 1.027$)$ & 0.417 & - & - \\
\hline Sex (M vs F) & $1.210(0.489$ to 2.991$)$ & 0.680 & - & - \\
\hline Motor phenotype at presentation $\ddagger$ & & 0.838 & - & - \\
\hline TD vs PIGD & $0.747(0.282$ to 1.976$)$ & 0.556 & - & - \\
\hline TD vs INT & $0.918(0.179$ to 4.722$)$ & 0.919 & - & - \\
\hline Average LED & $1.002(1.001$ to 1.003$)$ & 0.019 & $1.002(1.001$ to 1.003$)$ & 0.019 \\
\hline MAPT H1/H1 haplotype & 1.178 (0.698 to 1.990$)$ & 0.539 & - & - \\
\hline
\end{tabular}

For non-categorical covariates, RR represents change in risk per unit change in predictor variable.

*Estimated from National Adult Reading Test score.

†Dichotomised about the median value.

$\neq$ Phenotype defined according to algorithm of Zetusky et al.

HYS-3, Hoehn-Yahr stage 3; INT, intermediate; LED, levodopa equivalent dose in mg/day; MAPT, microtubule associated protein $\tau$ gene; PD, Parkinson's disease; PIGD, postural instability gait disorder; TD, tremor dominant. 


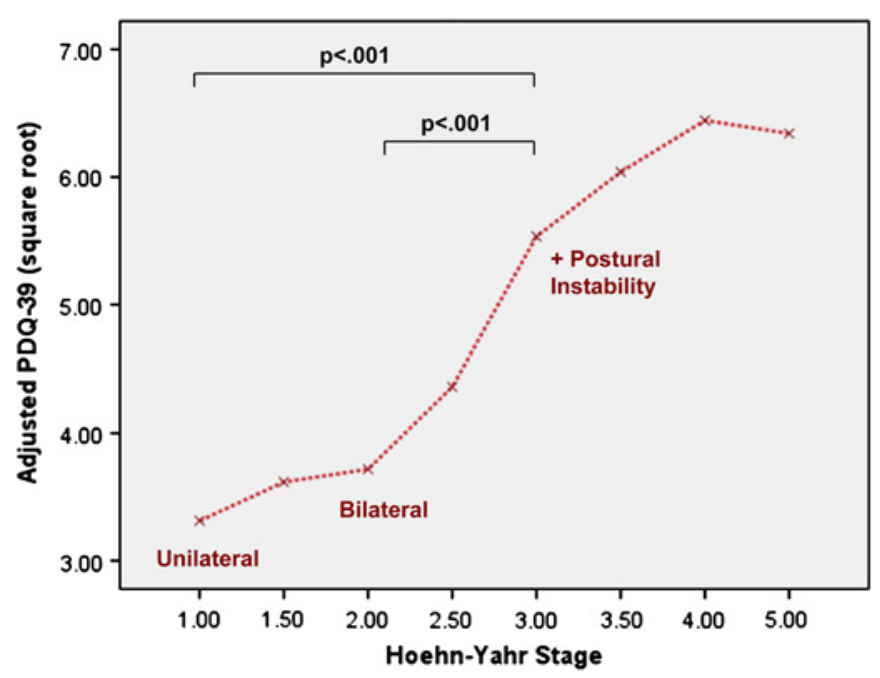

Figure 4 Estimated marginal means for quality of life (PDQ-39 scale) with advancing Hoehn-Yahr stage. ANCOVA has been used to adjust for the effects of age and disease duration. The results of post hoc comparisons between Hoehn-Yahr stage 3 and stages 1 and 2 are shown superimposed. PDQ-39 scores have been normalised by applying a square root transformation.

studies performed in hospital based cohorts at various times from diagnosis are biased towards capturing patients in better prognostic groups which most likely accounts for the very low progression rates reported in some studies of this type. Conversely, although data from the placebo arms of drug studies indicate high rates of clinical progression early in the disease course, the short follow-up periods employed in these studies seriously challenges the UPDRS as an index of disease evolution. ${ }^{28}$ Thus although our figure is closest to that of Schrag et al, ${ }^{5}$ we would argue that, given the representative nature of our cohort and the prolonged period of follow-up, our estimate is the most accurate yet produced. Furthermore, we have shown that by defining a subset of predominantly axial symptoms, an index of progression more sensitive than the overall UPDRS-3 score, hitherto the gold-standard, ${ }^{29}$ can be created. This is consistent with previous reports of increasing axial symptom burden in advanced $\mathrm{PD}^{30} 31$ and would suggest that modified rating scales specifically evaluating these symptoms may have clinimetric utility in PD. This is not something which has been specifically addressed in the recent revision of the UPDRS. ${ }^{32}$

HYS-3 is characterised by the onset of axial impairment and is associated with a step deterioration in QOL. Increased age at disease onset is a significant risk factor for progression to HYS-3, likely due to the synergistic effects of age dependent neuronal loss and parkinsonian neurodegeneration in the aged brain. ${ }^{33} \mathrm{We}$ recognise that the co-occurrence of other age related comorbidities which may independently affect balance, such as peripheral neuropathy or osteoarthritis, may also have contributed to this association. Although our analysis would suggest that presentation with a tremor dominant phenotype offers relative protection from progression, the relevant factor is probably a lower burden of axial symptoms in these patients rather than the presence of tremor.

A potential criticism of our study is that, as patients were consistently assessed in the 'on' state, we have measured variability in treatment response rather than true disease progression. We would argue that by assessing patients in the optimum clinical state, our data represent the best outcomes that can be achieved in practice with conventional treatment. In general, therapies purporting to modify the course of the disease should offer improvements over and above what can be achieved with best symptomatic therapy. Furthermore, there is evidence that levodopa itself has either a disease modifying effect or at least a prolonged symptomatic effect. ${ }^{34}$ Thus assessing medicated patients in the 'off' state is not the same as assessing never medicated patients; even symptomatic treatments may be altering the natural history of PD. Finally, the study of Clissold et al has previously indicated that, beyond 5 years of disease, 'on' and 'off' scores deteriorate in parallel. ${ }^{35}$

We used a linear model in our analysis of PD progression but there is evidence that the rate of neurodegeneration in PD may follow an exponential course, declining with time. ${ }^{36}$ However a previous study has shown that the trajectory of disease

Table 2 Reported rates of clinical progression in Parkinson's disease from published studies

\begin{tabular}{|c|c|c|c|c|c|c|}
\hline Study (year) & Design & No & Total FU & $\begin{array}{l}\text { Age at onset } \\
\text { (mean (SD)) }\end{array}$ & $\begin{array}{l}\text { PD duration at } \\
\text { entry (mean (SD)) }\end{array}$ & $\begin{array}{l}\text { UPDRS-3 change pa } \\
\text { (\% max) }\end{array}$ \\
\hline DATATOP $(1993)^{3}$ & CT & 199 & $6-21$ months & $61.1(9.5)$ & $<5$ years & $8.9(8.3 \%)$ \\
\hline Olanow et $a l^{2}$ & СТ & 40 & 14 months & $64.5(1.8)$ & $3.2(0.6)$ & $3.5(3.2 \%)$ \\
\hline Parks. Study Group (1996) ${ }^{1}$ & CT & 66 & $\leq 12$ months & $60.7(10.9)$ & $1.7(1.4)$ & $5.5(5.1 \%)$ \\
\hline Shults et al $(2002)^{47}$ & СТ & 16 & $\leq 16$ months & $63.1(12.1)$ & $<5$ years & $6.5(6.0 \%)$ \\
\hline ELLDOPA $(2004)^{34}$ & СТ & 90 & 42 weeks & $64.4(10.4)$ & $0.4(0.4)$ & $6.4(5.9 \%)$ \\
\hline ADAGIO $(2009)^{46}$ & СТ & 545 & 36 weeks & $61.9(9.7)$ & $0.4(0.4)$ & $7.3(4.1 \%)^{*}$ \\
\hline Louis et al $(1999)^{7}$ & $C, P$ & 237 & Up to 8 years & $72.8(10.5)$ & $6.8(6.8)$ & $1.5(1.4 \%)$ 'on' \\
\hline Goetz et al $(2000)^{6}$ & $\mathrm{H}, \mathrm{P}$ & 221 & Up to 4 years & $\begin{array}{l}56.7 \text { (HYS-2) } \\
59.3 \text { (HYS-3) }\end{array}$ & 1.17 (both groups) & $\begin{array}{l}0.1(0.1 \%) \text { (HYS-2) 'on' } \\
0.9(0.8 \%) \text { (HYS-3) 'on' }\end{array}$ \\
\hline Jankovic et al (2001) ${ }^{4}$ & $H, P$ & 297 & Up to 17 years & 55.1 (NR) & 6.5 (NR) & $\begin{array}{l}1.4(1.3 \%) \text { 'on' } \\
3.0(2.8 \%) \text { 'off' }\end{array}$ \\
\hline Alves et $a l^{8}$ & $H, P$ & 89 & 8 years & $59.4(9.7)$ & $8.8(4.8)$ & $3.3(3.1 \%)$ 'on' \\
\hline Schrag et al $(2007)^{5}$ & $H, P$ & 128 & 12 months & $57.6(12)$ & $9.3(7.4)$ & $3.3(3.1 \%)$ 'on' \\
\hline Harrison et al $(2009)^{48}$ & $\mathrm{H}, \mathrm{P}$ & 162 & 2.2 years (mean) & $58.2(11) \dagger$ & $7(6)$ & $0.3(0.3 \%)$ 'on' \\
\hline Present study & C, I & 122 & 5.5 years (mean) & $69.5(9.8)$ & From diagnosis & $2.24(2.1 \%)$ 'on' \\
\hline
\end{tabular}

The top panel displays results from the placebo arms of clinical trials, the lower panel results from longitudinal naturalistic studies.

${ }^{*}$ Rates of progression are estimated in the 'off' state unless otherwise indicated, and on the UPDRS-3 except for total UPDRS score.

†Data reported for a larger, cross sectional cohort of 888 cases within which the longitudinal cohort was nested.

C, community based; CT, clinical trial; FU, follow-up; H, hospital based; HYS-2, -3, Hoehn-Yahr stage 2, stage 3; I, incident; NR, not reported; P, prevalent; PD, Parkinson's disease; UPDRS Unified Parkinson's Disease Rating Scale. 
development measured on clinimetric scales does not differ significantly from linearity. ${ }^{37}$ As accurate estimates of progression rely on making repeated measures in the same patients, older patients, or those with more aggressive disease, are less likely to be available for follow-up which may lead to underestimation of the overall rate of progression. We have attempted to overcome this through the concomitant use of survival analysis, the outcome of which is less influenced by censored observations.

The mean time to dementia in our cohort was 6.2 years. This is lower than reported in a number of other series ${ }^{38}$ despite our cohort being community based and therefore having a relatively old age at disease onset. Dementia risk is associated with increased disease duration, ${ }^{39}$ and this lower figure is likely to reflect the fact that in the present study patients were followed from the point of diagnosis. We have shown that our previously reported association between the $\tau \mathrm{H} 1 / \mathrm{H} 1$ haplotype and dementia risk is maintained at follow-up of up to 7.9 years using statistically robust survival analysis. In a multivariate analysis, increased age and impaired performance on a task of semantic fluency, principally mediated by the temporal lobes, ${ }^{40}$ were associated with an increased risk of dementia, but not motor phenotype nor performance on other cognitive tasks at baseline. The $\mathrm{H} 1 / \mathrm{H} 1 \tau$ haplotype results in altered cortical $\tau$ transcription patterns $^{12}$ and, given the reported interaction between $\tau$ and $\alpha$-synuclein, ${ }^{41}$ may promote more rapid evolution of Lewy body pathology. The $\tau$ genotype had no influence on progression to HYS-3, probably because while the substrate of dementia in PD is generally believed to be cortical PD pathology, ${ }^{42}$ axial $\mathrm{PD}$ symptoms instead result from involvement of extranigral brainstem loci ${ }^{43}$ which may follow a slightly different pathogenic pathway.

Treatment induced dyskinesias were comparatively uncommon in our cohort and occurred at an estimated mean 6.6 years from diagnosis, in line with previous reports from community based series. ${ }^{44}$ Adjusting for disease duration, younger age at PD onset was not an independent risk factor for dyskinesia, and we conclude that it is more probable that increased cumulative exposure to dopaminergic therapy over the course of the illness accounts for the previously described association between age at onset and dyskinesia risk. ${ }^{45}$

Finally, we have reported only preliminary mortality data for this cohort. A more detailed analysis will form the basis of a future study, comprising in addition the results of clinicopathological analysis of subjects who have consented for post mortem brain donation.

\section{CONCLUSION}

Our study suggests that the natural history of treated PD is most accurately characterised by the insidious worsening of axial and postural symptoms, superimposed on which disease complications-'PD milestones'-occur (figure 5). These milestones, which may include the onset of gait freezing or levodopa induced dyskinesias, as well as the development of dementia and postural instability, are the major determinants of sufferers' experience of the disease. Hitherto, the design of trials of putative disease modifying agents has focused on the demonstration of sustained symptomatic effects (eg, Olanow et $a l^{46}$ ). Such a strategy may not, however, identify effects which translate into clinically meaningful benefits for patients. In our model, the target of such therapies is to shift the time to the major PD milestones into the future, an effect which can be regarded as true modulation of the natural history of the disease.

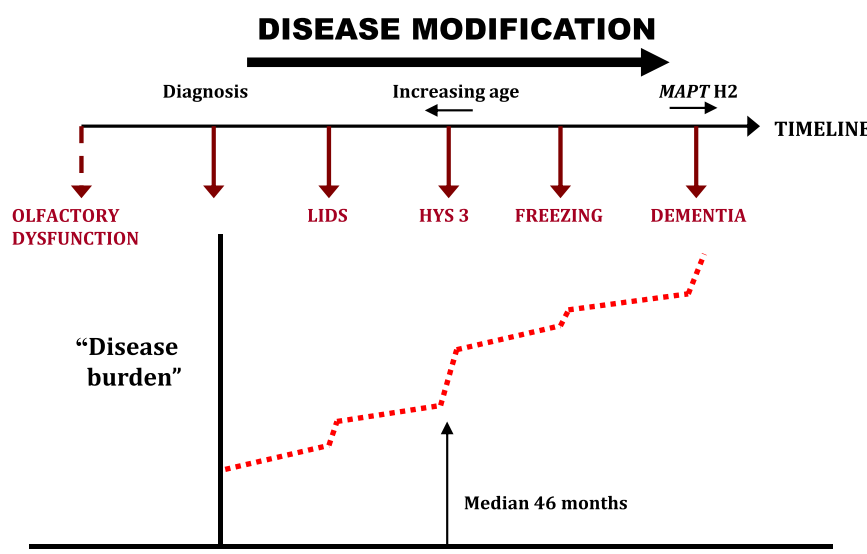

Figure 5 'Milestone model' of Parkinson's disease (PD) progression. The natural history of PD is here defined by the development of disease complications on a variable timeline from the point of diagnosis. Each of these milestones conveys a step worsening in the patient's experience of the disease. They can be shifted along the timeline through the influence of risk factors - for instance, increased age produces a 'left shift' in the time to Hoehn-Yahr stage 3 (HYS-3) onset, MAPT H2 carrier status a 'right shift' in the time to dementia. In this model, the efficacy of disease modifying therapies is evaluated in their ability to postpone the onset of these PD milestones. Olfactory dysfunction is included to illustrate that this concept can be extended to encompass premotor milestones. LIDS, levodopa induced dyskinesias; MAPT, (gene encoding) microtubule associated protein $\tau$.

In the absence of sensitive disease biomarkers, clinical measures of progression will remain a cornerstone of $\mathrm{PD}$ research. The existing strategies for measuring $\mathrm{PD}$ progression, namely the UPDRS (and presumably the MDS-UPDRS), may only capture aspects of disease evolution in treated patients. Our analysis of motor progression on both HYS and UPDRS converges on the emergence of axial symptoms-dysfunction in posture, gait and balance-in defining the natural history of treated PD. While improvements in clinimetric measurement of such features would have clear potential in evaluating disease progression, in the longer term there is a pressing unmet need to develop therapies specifically to target these features of the disease, and these will obviously encompass disease modifying therapies.

Acknowledgements The authors wish to thank Dr Stan Lazic for assistance with the statistical analysis.

Funding This work was supported by a National Institute of Health research award to Addenbrooke's Hospital and the University of Cambridge; the Cure Parkinson's Trust; and Parkinson's UK. JRE is supported by the Van Geest Foundation through a Gussy Marlowe Clinician Fellowship, and is in receipt of a Raymond and Beverley Sackler Studentship.

Competing interests None.

Ethics approval This study was conducted with the approval of the Cambridge 2 Local Research Ethics Committee.

Contributors JRE wrote the article, which was reviewed by all the listed authors. All authors contributed to the design of the study. SLM, CHW-G and TF collected the data. JRE performed the statistical analysis. Statistical methods were reviewed by RA Barker.

Provenance and peer review Not commissioned; externally peer reviewed.

\section{REFERENCES}

1. Anon. Effect of lazabemide on the progression of disability in early Parkinson's disease. The Parkinson Study Group. Ann Neurol 1996;40:99-107.

2. Olanow CW, Hauser RA, Gauger L, et al. The effect of deprenyl and levodopa on the progression of Parkinson's disease. Ann Neurol 1995;38:771-7. 
3. Anon. Effects of tocopherol and deprenyl on the progression of disability in early Parkinson's disease. The Parkinson Study Group. N Engl J Med 1993;328:176-83.

4. Jankovic J, Kapadia AS. Functional decline in Parkinson disease. Arch Neurol 2001:58:1611-15

5. Schrag A, Dodel R, Spottke A, et al. Rate of clinical progression in Parkinson's disease. A prospective study. Mov Disord 2007:22:938-45.

6. Goetz CG, Stebbins GT, Blasucci LM. Differential progression of motor impairment in levodopa-treated Parkinson's disease. Mov Disord 2000;15:479-84.

7. Louis ED, Tang MX, Cote L, et al. Progression of parkinsonian signs in Parkinson disease. Arch Neurol 1999;56:334-7.

8. Alves G, Wentzel-Larsen T, Aarsland D, et al. Progression of motor impairment and disability in Parkinson disease: a population-based study. Neurology 2005; $65: 1436-41$

9. Poewe W. The natural history of Parkinson's disease. J Neurol 2006;253(Suppl 7): VIII-6.

10. Goris A, Williams-Gray CH, Clark GR, et al. Tau and alpha-synuclein in susceptibility to, and dementia in, Parkinson's disease. Ann Neurol 2007:62:145-53.

11. Williams-Gray $\mathbf{C H}$, Foltynie T, Brayne $\mathrm{CE}$, et al. Evolution of cognitive dysfunction in an incident Parkinson's disease cohort. Brain 2007;130(Pt 7):1787-98.

12. Williams-Gray $\mathbf{C H}$, Evans JR, Goris $\mathrm{A}$, et al. The distinct cognitive syndromes of Parkinson's disease: 5 year follow-up of the CamPalGN cohort. Brain 2009;132 (Pt 11):2958-69.

13. Foltynie T, Brayne CE, Robbins TW, et al. The cognitive ability of an incident cohort of Parkinson's patients in the UK. The CamPaIGN study. Brain 2004;127 (Pt 3):550-60.

14. Gibb WR, Lees AJ. The relevance of the Lewy body to the pathogenesis of idiopathic Parkinson's disease. J Neurol Neurosurg Psychiatry 1988;51:745-52.

15. Brodsky MA, Godbold J, Roth T, et al. Sleepiness in Parkinson's disease: a controlled study. Mov Disord 2003;18:668-72.

16. Fahn SERL. Unified Parkinson's disease rating scale. In: Fahn SERL, Marsden CD, Goldstein M, et al, eds. Recent developments in parkinson's disease. New York: Macmillan Healthcare Information, 1987:153-63.

17. Movement Disorder Society Task Force on Rating Scales for Parkinson's Disease. The Unified Parkinson's Disease Rating Scale (UPDRS): status and recommendations. Mov Disord 2003;18:738-50.

18. Zetusky WJ, Jankovic J, Pirozzolo FJ. The heterogeneity of Parkinson's disease: clinical and prognostic implications. Neurology 1985;35:522-6.

19. Levy G, Louis ED, Cote L, et al. Contribution of aging to the severity of different motor signs in Parkinson disease. Arch Neurol 2005:62:467-72.

20. Hoehn MM, Yahr MD. Parkinsonism: onset, progression and mortality. Neurology 1967:17:427-42.

21. Goetz CG, Poewe W, Rascol 0, et al. Movement disorder society task force report on the Hoehn and Yahr staging scale: status and recommendations. Mov Disord 2004:19:1020-8.

22. Roos RA, Jongen JC, van der Velde EA. Clinical course of patients with idiopathic Parkinson's disease. Mov Disord 1996;11:236-42.

23. Jankovic J, McDermott M, Carter J, et al. Variable expression of Parkinson's disease: a base-line analysis of the DATATOP cohort. The Parkinson Study Group. Neurology 1990;40:1529-34.

24. Jenkinson C, Peto V, Harris R, et al. The Parkinson's Disease Questionnaire PDQ-39 User Manual. 2 edn. London: Oxford University Press, 2008.

25. Pinheiro JC, Bates DM. Mixed effects models in S and S-Plus. New York: Springer, 2000
26. Field A. Multilevel linear models. Discovering statistics using SPSS. 3 edn. London: Sage Publications Ltd, 2009.

27. Ala TA, Hughes LF, Kyrouac GA, et al. Pentagon copying is more impaired in dementia with Lewy bodies than in Alzheimer's disease. J Neurol Neurosurg Psychiatry 2001;70:483-8

28. Ahlskog JE, Uitti RJ. Rasagiline, Parkinson neuroprotection, and delayed-start trials: still no satisfaction? Neurology 2010;74:1143-8.

29. Schrag A, Spottke A, Quinn NP, et al. Comparative responsiveness of Parkinson's disease scales to change over time. Mov Disord 2009:24:813-18.

30. Bonnet AM, Loria Y, Saint-Hilaire MH, et al. Does long-term aggravation of Parkinson's disease result from nondopaminergic lesions? Neurology 1987:37:1539-42.

31. Hely MA, Morris JG, Reid WG, et al. Sydney multicenter study of parkinson's disease: non-L-dopa-responsive problems dominate at 15 years. Mov Disord 2005:20:190-9.

32. Goetz CG, Fahn S, Martinez-Martin P, et al. Movement Disorder Society-sponsored revision of the Unified Parkinson's Disease Rating Scale (MDS-UPDRS): process, format, and clinimetric testing plan. Mov Disord 2007;22:41-7.

33. Levy G. The relationship of Parkinson disease with aging. Arch Neurol 2007:64:1242-6

34. Fahn S, Oakes D, Shoulson I, et al. Levodopa and the progression of Parkinson's disease. N Engl J Med 2004;351:2498-508.

35. Clissold BG, McColl CD, Reardon KR, et al. Longitudinal study of the motor response to levodopa in Parkinson's disease. Mov Disord 2006;21:2116-21.

36. Maetzler W, Liepelt I, Berg D. Progression of Parkinson's disease in the clinical phase: potential markers. Lancet Neurol 2009:8:1158-71.

37. Schulzer M, Lee CS, Mak EK, et al. A mathematical model of pathogenesis in idiopathic parkinsonism. Brain 1994:117(Pt 3):509-16.

38. Aarsland D, Kurz MW. The epidemiology of dementia associated with Parkinson disease. J Neurol Sci 2010;289:18-22.

39. Hely MA, Reid WG, Adena MA, et al. The Sydney multicenter study of Parkinson's disease: the inevitability of dementia at 20 years. Mov Disord 2008:23:837-44

40. Henry JD, Crawford JR. A meta-analytic review of verbal fluency performance following focal cortical lesions. Neuropsychology 2004;18:284-95.

41. Giasson BI, Forman MS, Higuchi M, et al. Initiation and synergistic fibrillization of tau and alpha-synuclein. Science 2003;300:636-40.

42. Aarsland D, Perry R, Brown A, et al. Neuropathology of dementia in Parkinson's disease: a prospective, community-based study. Ann Neurol 2005:58:773-6.

43. Pahapill PA, Lozano AM. The pedunculopontine nucleus and Parkinson's disease. Brain 2000;123(Pt 9):1767-83.

44. Schrag A, Quinn N. Dyskinesias and motor fluctuations in Parkinson's disease. A community-based study. Brain 2000;123(Pt 11):2297-305.

45. Schrag A, Hovris A, Morley D, et al. Young- versus older-onset Parkinson's disease: impact of disease and psychosocial consequences. Mov Disord 2003;18:1250-6.

46. Olanow CW, Rascol 0, Hauser R, et al. A double-blind, delayed-start trial of rasagiline in Parkinson's disease. N Engl J Med 2009:361:1268-78.

47. Shults CW, Oakes D, Kieburtz K, et al. Effects of coenzyme 010 in early Parkinson disease: evidence of slowing of the functional decline. Arch Neurol 2002:59:1541-50.

48. Harrison MB, Wylie SA, Frysinger RC, et al. UPDRS activity of daily living score as a marker of Parkinson's disease progression. Mov Disord 2009:24:224-30. 